\title{
Hot C-rich white dwarfs: testing the DB-DQ transition through pulsations
}

\author{
A. H. Córsico ${ }^{1,2, \star}$, A. D. Romero ${ }^{1,2, \star \star}$, L. G. Althaus ${ }^{1,3, \star}$, and E. García-Berro ${ }^{3,4}$ \\ 1 Facultad de Ciencias Astronómicas y Geofísicas, Universidad Nacional de La Plata, Paseo del Bosque S/N, (1900) \\ La Plata, Argentina \\ 2 Instituto de Astrofísica La Plata, IALP, CONICET-UNLP, Argentina \\ ${ }^{3}$ Departament de Física Aplicada, Escola Politècnica Superior de Castelldefels, Universitat Politècnica de Catalunya, \\ Av. del Canal Olímpic, s/n, 08860 Castelldefels, Spain \\ 4 Institut d'Estudis Espacials de Catalunya, c/Gran Capità 2-4, Edif. Nexus 104, 08034 Barcelona, Spain \\ e-mail: acorsico,aromero,althaus@fcaglp.unlp.edu.ar; garcia@fa.upc.edu
}

Received 13 May 2009 / Accepted 16 July 2009

\begin{abstract}
Context. Hot DQ white dwarfs are a new class of white dwarf stars that were discovered recently within the framework of the SDSS project. There are nine known hot DQ stars, out of a total of several thousands white dwarfs spectroscopically identified. Three hot DQ white dwarfs have been reported to exhibit photometric variability with periods compatible with pulsation $g$-modes.

Aims. We present a nonadiabatic pulsation analysis of carbon-rich hot DQ white dwarf stars. One of our main aims is to test the convective-mixing scenario for the origin of hot DQs by studying their pulsational properties.

Methods. Our pulsation study is based on the full evolutionary models of hot DQ white dwarfs developed by Althaus and collaborators, which consistently cover the entire evolution from the born-again stage to the white dwarf cooling track. Specifically, we present a stability analysis of white dwarf models from stages before the blue edge of the DBV instability strip $\left(T_{\text {eff }} \approx 30000 \mathrm{~K}\right)$, until the domain of the hot DQ white dwarfs (18000-24000 K), including the transition DB $\rightarrow$ hot DQ white dwarf. We explore evolutionary models with $M_{*}=0.585 M_{\odot}$ and $M_{*}=0.87 M_{\odot}$, and two values of the thickness of the He-rich envelope $\left(M_{\mathrm{He}}=2 \times 10^{-7} M_{*}\right.$ and $\left.M_{\mathrm{He}}=10^{-8} M_{*}\right)$. These envelopes are 4-5 orders of magnitude thinner than those of standard DB white dwarf models resulting from canonical stellar evolution computations.

Results. We found that at evolutionary phases in which the models are characterized by He-dominated atmospheres, they exhibit unstable $g$-mode pulsations typical of DBV stars, and when the models become DQ white dwarfs with carbon-dominated atmospheres, they continue being pulsationally unstable with characteristics similar to DB models, and in agreement with the periods detected in variable hot DQ white dwarfs. In particular, for models with $M_{\mathrm{He}}=10^{-8} M_{*}$, a narrow gap exists separating the DB from the DQ instability domains.

Conclusions. Our calculations provide strong support for the convective-mixing picture of the formation of hot DQs. In particular, our results suggest the existence of pulsating DB white dwarfs with very thin He-rich envelopes, which after passing the DBV instability strip become variable hot DQ stars. The existence of these DB stars with very thin envelopes should be investigated by asteroseismology.
\end{abstract}

Key words. stars: evolution - stars: interiors - stars: oscillations - white dwarfs

\section{Introduction}

The recent years have witnessed a great deal of work in the field of white dwarf stars. A recent example is the unexpected discovery of a population of white dwarfs characterized by carbondominated atmospheres at effective temperatures of between $\sim 18000$ and $\sim 24000 \mathrm{~K}-$ also known as hot DQ white dwarfs (Dufour et al. 2007). This finding attracted the attention of many researchers because the existence of these stars challenges the theory of stellar evolution, and could be indicative of the existence a new evolutionary channel of white-dwarf formation.

Dufour et al. (2008) proposed an evolutionary scenario to explain the origin of hot DQ white dwarfs. In this scenario,

* Member of the Carrera del Investigador Científico y Tecnológico, CONICET, Argentina.

$\star \star$ Fellow of CONICET, Argentina. undetected amounts of $\mathrm{He}\left(M_{\mathrm{He}} \lesssim 10^{-14} M_{\odot}\right)$ remaining in the C- and O-rich outer layers of a PG1159 star like the exotic object H1504+65, would be forced to float to the surface because of gravitational separation, leading to a He-dominated white dwarf, first of the spectral class DO and later of the spectral class DB. In this picture, a C-rich atmosphere should eventually emerge as the result of convective mixing at lower effective temperatures. The first quantitative assessment of this diffusive/convective mixing scenario was presented by Althaus et al. (2009). Using full evolutionary models that consistently cover the evolution from the born-again stage to the white dwarf cooling track, these authors presented strong theoretical evidence supporting this picture for the formation of hot DQs and the existence of an evolutionary link between these stars and the PG1159 stars, including H1504+65. It is worth mentioning that 
the models of Althaus et al. (2009) are characterized by a $\mathrm{He}$ content ranging from $M_{\mathrm{He}} \sim 10^{-7} M_{\odot}$ to $10^{-8} M_{\odot}$.

Even when the impact of the discovery of DQ white dwarfs had not declined, Montgomery et al. (2008) reported the finding of the first variable hot DQ star, SDSS J142625.70+575218.4 (with $\log g \sim 9$ and $T_{\text {eff }} \sim 19800 \mathrm{~K}$ ), with a confirmed period $\Pi \approx 418$ s. Shortly after, Barlow et al. (2008) reported the discovery of two additional variable hot DQ stars, SDSS J220029.08-074121.5 $\left(\log g \sim 8, T_{\text {eff }} \sim 21240 \mathrm{~K}\right)$ and SDSS J234843.30-094245.3 ( $\left.\log g \sim 8, T_{\text {eff }} \sim 21550 \mathrm{~K}\right)$, with periods $\Pi \approx 656 \mathrm{~s}$ and $\Pi \approx 1052 \mathrm{~s}$, respectively. The measured periodicities were interpreted as nonradial $g$-mode pulsations, similar to the well-studied pulsations of the GW Vir, V777 Her, and ZZ Ceti classes of white-dwarf variables. The pulsation hypothesis, however, was defied by the possibility that these stars could be AM CVn systems, because of the similarity exhibited in the pulse shape of the light curves (Montgomery et al. 2008). On the other hand, a compelling argument against the interacting binary hypothesis is that it does not explain why all hot DQ white dwarfs are grouped within the same range of temperatures, and none at higher or lower effective temperatures (Dufour et al. 2009). Ultimately, the pulsating nature of the variable hot DQ white dwarfs could be confirmed by the discovery of multiperiodicity. Following this line of reasoning, Fontaine et al. (2009) announced the discovery of an additional period $\Pi \sim 319 \mathrm{~s}$ (apart from the already known at $418 \mathrm{~s}$ ) in SDSS $\mathrm{J} 142625.70+575218.4$.

The origin of variability in hot DQ stars was addressed theoretically by Fontaine et al. (2008), who studied the hypothesis that the variability could be caused by pulsations. Their full nonadiabatic analysis found that $g$-modes can be excited in the range of temperature where real DQs are found (below $\sim 21500 \mathrm{~K}$ ) only if the models are characterized by substantial amounts of $\mathrm{He}\left(X_{\mathrm{He}} \gtrsim 0.25\right)$ in their C-rich envelopes. Dufour et al. (2008) estimated the $T_{\text {eff }}, \log g$, and $\mathrm{C} / \mathrm{He}$ ratio of the nine known hot DQ stars and constructed a dedicated stellar model for each object using the same modeling as in Fontaine et al. (2008). By using a nonadiabatic approach, Dufour et al. (2008) predicted that only SDSS J1426+5752 should exhibit pulsations, and failed to predict variability in SDSS J220029.08-074121.5 and SDSS J234843.30-094245.3. However, it appears that the pulsation models of Fontaine et al. (2008) and Dufour et al. (2008) are not entirely consistent with their proposed evolutionary picture for the formation of hot DQs. Indeed, the background models they assumed for their stability calculations are characterized by a He content several orders of magnitude higher than the content of He required by their evolutionary scenario to work (Dufour et al. 2008). In fact, the bottom of the He-dominated envelope in their stellar models is located at a fractional mass depth of $\log q_{\text {env }} \equiv \log \left(1-M_{r} / M_{*}\right)=-3$ (Fontaine et al. 2008).

In this work, we perform a nonadiabatic analysis of the pulsations of hot DQ white dwarfs on the basis of the full evolutionary models developed by Althaus et al. (2009). The models consistently cover the entire evolution from the born-again stage to the hot DQ white dwarf domain. As shown below, we are able to obtain a pulsational picture of the variable hot DQ white dwarfs that is entirely consistent with the diffusive/convective mixing evolutionary scenario proposed for their formation. The paper is organized as follows. In Sect. 2, we briefly describe some aspects of the stellar evolution and pulsation modeling that we employ in the present work. Section 3 describes the diffusive/convectivemixing scenario. We elaborate on our stability analysis in Sect. 4. Finally, Sect. 5 summarizes our results.

\section{Stellar and pulsation modeling}

The stellar models employed in this work were generated with the LPCODE stellar evolutionary code employed in our previous study of the formation of H-deficient post-AGB stars by a bornagain episode (see Althaus et al. (2005) and Miller Bertolami \& Althaus (2006) for details about the code). The code is specifically designed to compute the formation and evolution of white dwarf stars. In LPCODE, special emphasis is given to the treatment of changing the chemical abundances, including diffusive overshooting and non-instantaneous mixing, which are of primary importance in the calculation of the thermal pulses and born-again stage that lead to the formation of PG1159 stars. Of relevance to the present study, we mention that convection is treated within the formalism of the mixing length theory given by the ML2 parametrization (Tassoul et al. 1990). We note that this parametrization leads to a theoretical blue edge of the DB instability strip consistent with observations (Beauchamp et al. 1999; Córsico et al. 2009).

The white dwarf evolutionary sequences employed in our stability study correspond to those developed in Althaus et al. (2009) to explore the formation of hot DQs via the diffusive/convective mixing scenario. The initial stellar models for those sequences are realistic PG1159 stellar configurations derived from the full evolution of their progenitor stars (Miller Bertolami \& Althaus 2006). In particular, we considered sequences with stellar masses of 0.87 and $0.585 M_{\odot}$. The chemical stratification of the initial models consists of a $\mathrm{CO}$ core, which is the result of core He burning in prior stages, surrounded by a He-, C- and O-rich envelope, in agreement to what is observed in PG1159 stars. The fractional mass of the He-rich envelope is in the range $10^{-8} \leq M_{\mathrm{He}} / M_{*} \leq 2 \times 10^{-7}$. The sequence with $M_{*}=0.87 M_{\odot}$ was specifically computed by Althaus et al. (2009) to explore the evolutionary connection between H1504+65 and hot DQ white dwarfs. For this stellar mass, the He content of $2 \times 10^{-7} M_{*}$ corresponds to the maximum He content expected in $\mathrm{H} 1504+65$, if we assume a post-born-again origin for this star (Miller Bertolami \& Althaus 2006). The evolutionary calculations were computed from $T_{\text {eff }}=100000 \mathrm{~K}$ down to the domain of effective temperatures typical of hot DQs ( 20 000-17000 K).

The pulsation stability analysis was performed with the finite-difference nonadiabatic pulsation code described in Córsico et al. (2006). The code solves the full sixth-order complex system of linearized equations and boundary conditions as given by Unno et al. (1989). Our code provides the dimensionless complex eigenvalue $(\omega)$ and eigenfunctions according to the formulation given in Unno et al. (1989). Nonadiabatic pulsation periods and normalized growth rates are evaluated to be $\Pi=2 \pi / \mathfrak{R}(\sigma)$ and $\eta=-\mathfrak{J}(\sigma) / \mathfrak{R}(\sigma)$, respectively. Here, $\mathfrak{R}(\sigma)$ and $\mathfrak{J}(\sigma)$ are the real and the imaginary part, respectively, of the complex eigenfrequency $\sigma=\left(G M_{*} / R^{3}\right)^{1 / 2} \omega$. Our code also computes the differential work function, $\mathrm{d} W(r) / \mathrm{d} r$, and the running work integral, $W(r)$, as in Lee \& Bradley (1993). These functions are extremely useful for determining the driving and damping regions of the stellar models. As in Fontaine et al. (2008) and Dufour et al. (2008), our nonadiabatic computations rely on the frozen-convection approximation, in which the perturbation of the convective flux is neglected. While this approximation is known to give unrealistic locations of the red edge of instability, it leads to satisfactory predictions for the location of the blue edge of the ZZ Ceti (DAV) instability strip - see Brassard \& Fontaine (1999) - and also for the V777 Her (DBV) 


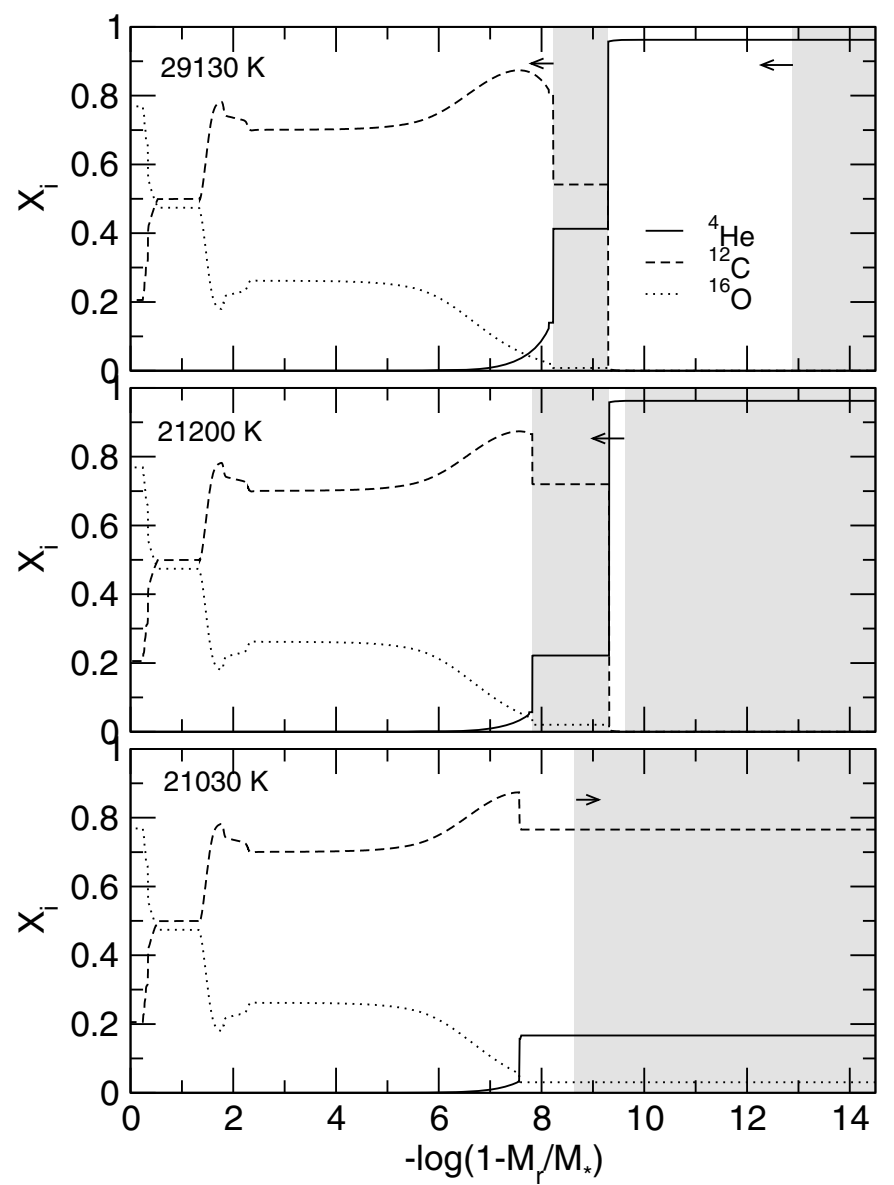

Fig. 1. The internal chemical abundances of helium, carbon, and oxygen for models with $M_{*}=0.585 M_{\odot}$ and $M_{\mathrm{He}}=10^{-8} M_{*}$ and effective temperatures (from top to bottom) of $\sim 29100,21200$, and $21030 \mathrm{~K}$. The gray regions display convective zones.

instability strip - see Beauchamp et al. (1999) and, more recently, Córsico et al. (2009).

\section{Evolutionary results: the diffusive/convective mixing scenario}

We briefly summarize the diffusive/convective mixing picture developed by Althaus et al. (2009) to explain the origin of the hot DQs. In this scenario, the starting models are realistic PG1159 stellar configurations with small contents of residual $\mathrm{He}$, the fractional masses being in the range $10^{-8} \lesssim M_{\mathrm{He}} / M_{*} \lesssim$ $2 \times 10^{-7}$. At the beginning of the hot phase of white-dwarf evolution, the chemical abundance distribution of the envelope of PG1159 stars is drastically modified by gravitational settling that forces $\mathrm{He}$ to float to the surface and heavier elements $(\mathrm{C}$ and $\mathrm{O}$ ) to sink. As a result, the star becomes a DO white dwarf with a very thin He-rich envelope. With further cooling, the star reaches the domain of DB white dwarfs $\left(T_{\text {eff }} \sim 30000 \mathrm{~K}\right)$. The upper panel of Fig. 1 shows the internal abundance distribution of ${ }^{4} \mathrm{He},{ }^{12} \mathrm{C}$, and ${ }^{16} \mathrm{O}$ for a selected model with $M_{*}=0.585 M_{\odot}$, $M_{\mathrm{He}}=10^{-8} M_{*}$, and $T_{\text {eff }}=29130 \mathrm{~K}$. We note that, at this effective temperature, the DB model star is characterized by an inward-growing outer He convection zone (gray region) produced by the recombination of HeII. In addition, there is a convective intershell region at $8.2 \lessgtr-\log \left(1-M_{r} / M_{*}\right) \lessgtr 9.3$. The existence of this convection zone is the consequence of the large opacity of the C-enriched layers below the pure He envelope. As the model star cools, the bottom of this convection region moves inwards, thus increasing the $\mathrm{C}$ abundance there, as shown in the middle panel of Fig. 1, which corresponds to a DB model at $T_{\text {eff }}=21200 \mathrm{~K}$. At this stage, the bottom of the outer convection region is very close to the upper boundary of the intershell convective region. Shortly afterwards, both convection zones eventually merge, and a very efficient mixing episode - favoured by the presence of the intershell convection zone - leads to an envelope rich in $\mathrm{C}$ and $\mathrm{He}\left(X_{\mathrm{C}}=0.77, X_{\mathrm{He}}=0.17\right)$, with a trace abundance of $\mathrm{O}\left(X_{\mathrm{O}}=0.03\right)$. As a result, the DB white dwarf model appears as a hot DQ white dwarf. The chemical abundance distribution at this stage is depicted in the bottom panel of Fig. $1\left(T_{\text {eff }}=21030 \mathrm{~K}\right)$.

The evolution following the merger of the convection zones is difficult to model. In fact, once the C-rich convective envelope has developed, it is expected a substantial depletion of carbon in the whole convective envelope. Indeed, the diffusion timescale at the base of the convection zone of the newly formed DQ is about $10^{5}-10^{6} \mathrm{yr}$, substantially shorter than the cooling timescale, of about $10^{8}$ yr. Hence, we expect that the hot DQ stage is indeed a short-lived phase, after which the star should recover its identity as a He-atmosphere DB white dwarf quickly. The picture is even more complicated because the change in composition in the convective envelope will change the depth of the convection zone, which will affect, in turn, the timescales of diffusion. Our numerical treatment of diffusion does not allow us to follow properly this feedback between convection and diffusion. We do not exclude the possibility that the star experiences additional mixing episodes, eventually reaching some stationary situation below $T_{\text {eff }} \sim 13000 \mathrm{~K}$ in which the star would be a He-rich DB white dwarf with traces of $\mathrm{C}$, that is, a DQ white dwarf such as those observed at these effective temperatures (see, e.g., Bergeron et al. 2001). Additional computations would be needed to test this possibility.

The evolution described above is qualitatively similar to that of the remaining sequences analyzed here. However, the effective temperature at which the transition from DB to DQ white dwarf occurs, and the final surface chemical abundances, vary for different He contents and different stellar masses. For instance, in the case of the sequence with $M_{*}=0.585 M_{\odot}$ and $M_{\mathrm{He}}=2 \times 10^{-7} M_{*}$, the transition occurs at $T_{\text {eff }} \sim 19500 \mathrm{~K}$, an effective temperature somewhat lower than when the He content is 20 times lower $\left(T_{\text {eff }} \sim 21000 \mathrm{~K}\right)$. On the other hand, the final abundance of $\mathrm{C}$ at the surface is markedly lower for higher He contents. This trend is in line with observations (Dufour et al. 2008). If we consider more massive models and a fixed value of $M_{\mathrm{He}}$, the transition from DB to DQ occurs at somewhat lower effective temperatures (Althaus et al. 2009). To help clarify this point, we show in Fig. 2 the evolution of the convection zones in terms of the effective temperature for the two values of both the stellar mass and He content analyzed in this paper. We note that for effective temperatures below $23000 \mathrm{~K}$, the more massive sequence is characterized by deeper He convection envelopes. However, for temperatures less than about $21000 \mathrm{~K}$, this trend is reversed and the less massive sequence is characterized by a deeper He convection envelope. At this effective temperature, the base of the He convection zone is close to the outer edge of the convective intershell region in the sequence with $M_{\mathrm{He}}=10^{-8} M_{*}$, and so we expect that the DQ is formed at about the same effective temperature in both stellar masses. For the sequence with $M_{\mathrm{He}}=2 \times 10^{-7} M_{*}$, clearly the DQ formation is expected at a substantially lower effective temperature in the more massive model. We also note that the rate of the inward increase of the base of the He convection zone by 

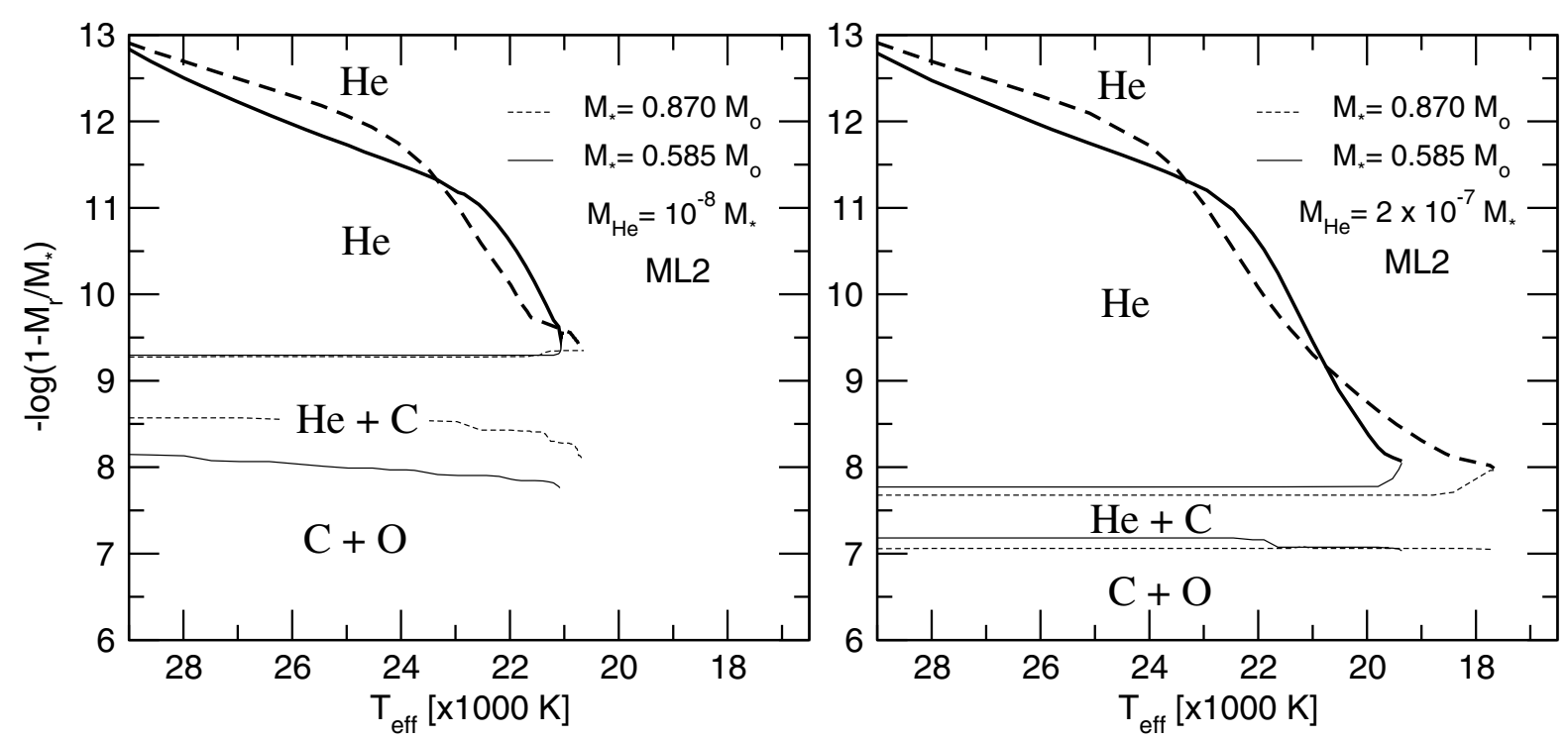

Fig. 2. The location of the boundaries of the convective regions in terms of the effective temperature. Left (right) panel corresponds to the sequence with $M_{\mathrm{He}}=10^{-8} M_{*}\left(M_{\mathrm{He}}=2 \times 10^{-7} M_{*}\right)$. Solid (dashed) lines correspond to the sequence with $M_{*}=0.585 M_{\odot}\left(M_{*}=0.870 M_{\odot}\right)$. Thick lines are associated to the location of the base of the outer convection zone, whereas thin lines depict the location of the boundaries of the convective carbon intershell. Stages after the merger of the two convection zones are not shown.

the time it approaches the convective carbon intershell, is lower. This is because of the presence of traces of carbon left by chemical diffusion. The presence of trace carbon just above the outer edge of the convective intershell region causes larger radiative opacities, larger temperature gradients and eventually shallower convection zones, compared to the behavior expected in pure $\mathrm{He}$ envelopes.

Hitherto, we have described in some detail the diffusive/convective mixing scenario that is able to explain the formation of the hot DQ white dwarfs from DB white dwarfs with thin He-rich envelopes. At present, observations strongly indicate that DB white dwarfs exhibit pulsations at effective temperatures between $23000 \mathrm{~K}$ and $28000 \mathrm{~K}$ (the DBV instability strip), and that DQ white dwarfs show photometric variations at $T_{\mathrm{eff}} \approx 20000 \mathrm{~K}$. It is unclear whether the diffusive/convective mixing picture can also predict the existence of the DB instability strip and the DQ instability domain at the effective temperatures required by the observations. In the next section, we perform a stability analysis that helps us to answer this question.

\section{Stability calculations}

We present a detailed pulsation stability analysis of our set of DB and DQ white dwarf models. As mentioned, numerical difficulties prevented us from any additional following evolution of our DQ sequences far beyond the transition from a DB to DQ white dwarf. Thus, we are restricted to studying mainly the location of the blue edge of the DQ instability domain, its dependence on both stellar mass and the content of $\mathrm{He}$, and the stability properties of DQ models located near this blue edge. This study of these important properties of variable DQ white dwarfs in terms of a self-consistent evolutionary picture is very timely and relevant.

We analyzed the pulsational stability of about 800 stellar models covering a wide range of effective temperatures $\left(30000 \gtrsim T_{\text {eff }} \gtrsim 18000 \mathrm{~K}\right)$ and stellar masses of $M_{*}=0.585 M_{\odot}$ and $M_{*}=0.870 M_{\odot}$. For each mass value, we considered two values of the He content: $M_{\mathrm{He}} / M_{*}=2 \times 10^{-7}$ and $M_{\mathrm{He}} / M_{*}=$ $10^{-8}$. For each stellar model, we studied the stability of $\ell=1$ $g$-modes with periods in the range $50 \mathrm{~s} \lesssim \Pi \lesssim 3000$ s, thus comfortably embracing the full period spectrum observed in variable DB and DQ stars.

\subsection{Template models}

We discuss the stability properties of two template white dwarf models selected from the evolutionary sequence with $M_{*}=$ $0.585 M_{\odot}$ and $M_{\mathrm{He}} / M_{*}=10^{-8}$. One of these models is a DB white dwarf model $\left(T_{\text {eff }}=24200 \mathrm{~K}\right)$, and the other is a hot DQ white dwarf model $\left(T_{\text {eff }}=21135 \mathrm{~K}\right)$. The normalized growth rate $(\eta)$ in terms of pulsation periods (П) for overstable $\ell=1$ modes corresponding to these models is shown in Fig. 3 . We note that modes excited in the DB model have pulsation periods in the range $650 \lesssim \Pi \lesssim 2000 \mathrm{~s}$, substantially longer than those excited in the DQ model $(200 \lesssim \Pi \lesssim 1000 \mathrm{~s})$. For the DB model, $\eta$ reaches a strong maximum at a period of about $1250 \mathrm{~s}$. On average, for this model the excitation is stronger for modes characterized by short periods, although at the edges of the instability band the value of $\eta$ notably drops. On the other hand, for the DQ model the modes with longer periods are more favoured for excitation, the growth rate for the shortest periods being more than seven orders of magnitude lower than for the modes with longer periods. We note that, for modes with the shortest periods, the value of $\eta$ is so small $\left(\lesssim 10^{-9}\right)$ that they are only marginally unstable. Hence, hereafter we consider a mode to be unstable if $\eta \gtrsim 10^{-9}$.

As clearly emphasized in Fig. 3, the $g$-mode excitation is noticeably stronger for the DB model than for the DQ model. Thus, the question arises about what would be the chance for a given excited mode in a DQ star to have time enough for developing observable amplitudes. In this respect, we note that for our template DQ model, the maximum and minimum $e$-folding times of the set of excited modes are $\tau_{\mathrm{e}}^{\max }=459 \mathrm{yr}(k=4$, $\left.\Pi=280.5 \mathrm{~s}, \eta=3.1 \times 10^{-9}\right)$ and $\tau_{\mathrm{e}}^{\min }=5 \mathrm{yr}(k=18, \Pi=847 \mathrm{~s}$, $\left.\eta=9 \times 10^{-7}\right)$, respectively ${ }^{1}$. These times are by far shorter than

${ }^{1}$ The $e$-folding times are defined to be $\tau_{\mathrm{e}} \equiv 1 /|\mathfrak{I}(\sigma)|$, such that the time dependence of the amplitude of the pulsations is given by $\xi(t) \propto$ $\mathrm{e}^{i \sigma t}$, and $\mathfrak{J}(\sigma)<0$ for overstable modes. 


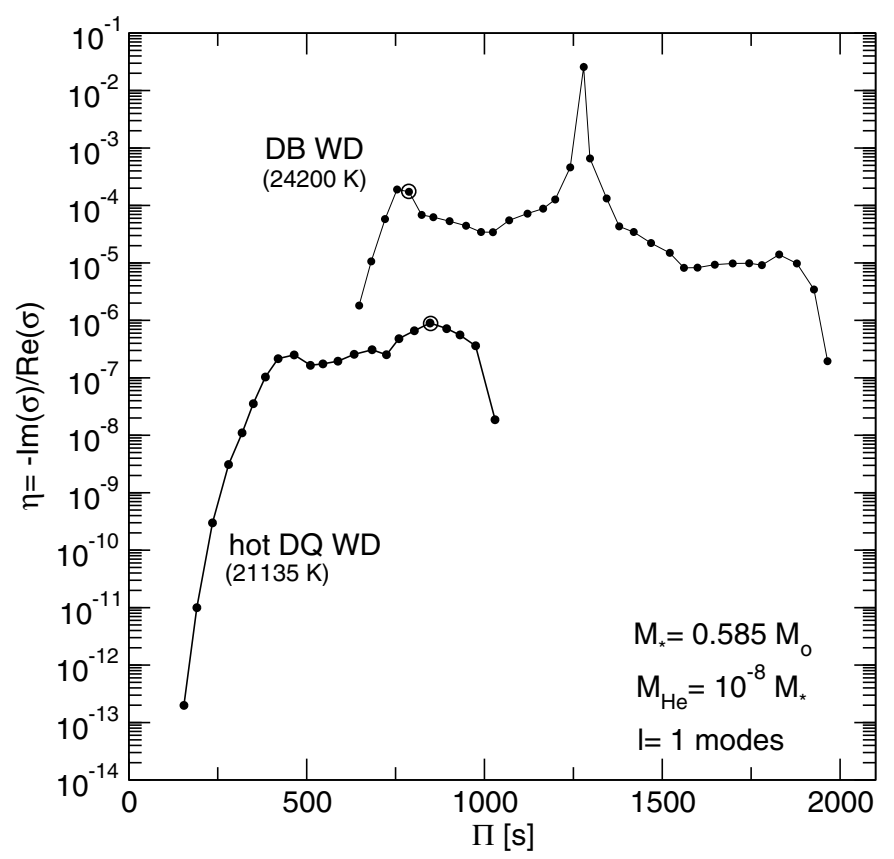

Fig. 3. The normalized growth rate in terms of period (in seconds) for overstable $g$-modes corresponding to a DB model $\left(T_{\text {eff }}=24200 \mathrm{~K}\right)$ and a hot DQ white dwarf model $\left(T_{\text {eff }}=21135 \mathrm{~K}\right)$, both models corresponding to the same evolutionary sequence $\left(M_{*}=0.585 M_{\odot}\right.$ and $\left.M_{\mathrm{He}}=10^{-8} M_{*}\right)$. For each model, the circumscribed dot corresponds to the $k=18 g$-mode.

the evolutionary timescale, which ranges from $10^{7}$ to $10^{8} \mathrm{yr}$. This suggests that the excited modes in hot DQ white dwarfs should have sufficient time to reach observable amplitudes. It is worth mentioning that we arrive at the same conclusion as Fontaine et al. (2008), who use a set of DQ models and a nonadiabatic pulsation code that are completely independent from of ours.

Figure 4 illustrates the driving/damping process of our DB template model. The thick solid line corresponds to $\mathrm{d} W / \mathrm{d} r$ for an unstable mode with $k=18, \Pi=787 \mathrm{~s}$, and $\eta=1.7 \times 10^{-4}$ (marked as a circumscribed dot in the upper curve of Fig. 3). We also plot the logarithm of the Rosseland opacity, $\kappa$, and its logarithmic derivative, $\kappa_{\mathrm{T}} \equiv(\partial \log \kappa / \partial \log T)_{\rho}$. As can be seen, the region that destabilizes the mode (where $\mathrm{d} W / \mathrm{d} r>0$ ) is located in a region slightly below to the corresponding to the bump in the opacity at $-\log \left(1-M_{r} / M_{*}\right) \sim 12.5\left(T=1.32 \times 10^{5} \mathrm{~K}\right)$, close the base of the outer convection zone. This bump corresponds to the partial ionization of HeII. Above and below the driving region, there are two zones of damping. Since the contributions to driving largely overcome the damping effects, the $k=18$ mode is globally excited.

We also note the presence of a second bump in the opacity at $-\log \left(1-M_{r} / M_{*}\right) \sim 9\left(T=1.13 \times 10^{6} \mathrm{~K}\right)$, caused by the partial ionization of $\mathrm{CV}$ and CVI. Notably, this opacity bump does not contribute at all to the destabilization of the $k=18$ mode. So, in terms of pulsation stability, the mode does not "feel" the presence of the C-bump. For illustrative purposes, we included in Fig. 4 the logarithm of the Rosseland opacity (thin dot-dashed line) corresponding to a DB white dwarf model of the same total mass and effective temperature as our DB template model, but characterized by a thick He-rich envelope. This model was extracted from the calculations of Córsico et al. (2009). The run of the opacity is coincident with that of our DB template model, except at the location of the C-bump, because the thick-envelope DB model has no $\mathrm{C}$ in that region.

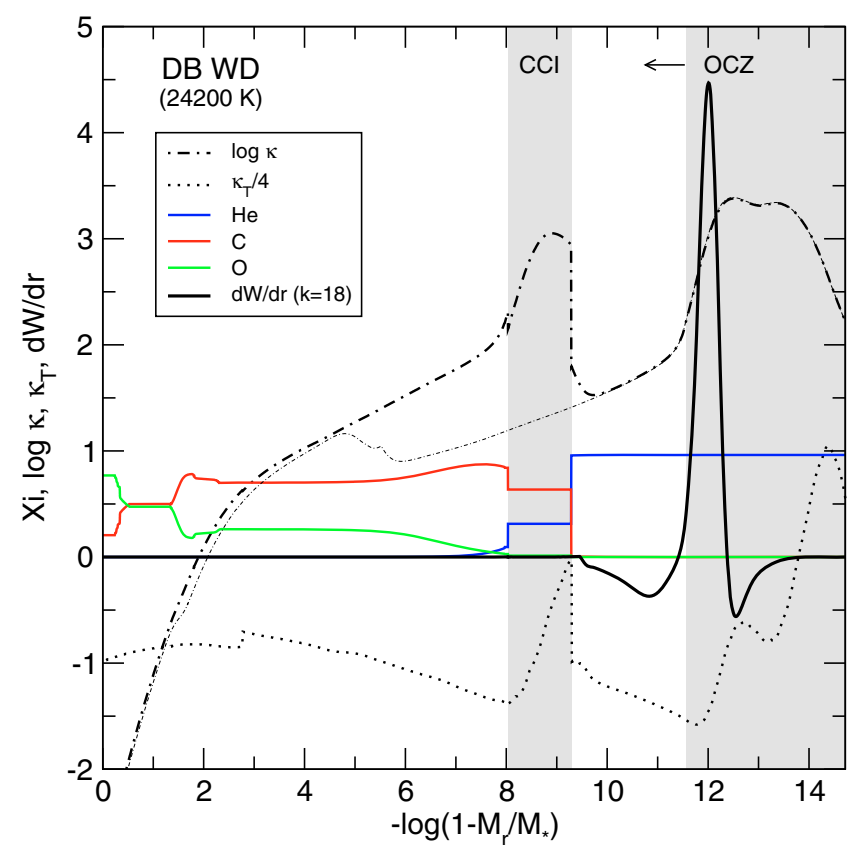

Fig. 4. The logarithm of the Rosseland opacity (dot-dashed line) the opacity logarithmic derivative $\left(\kappa_{\mathrm{T}}\right)$ (dotted line), and the differential work function (solid line) for a selected overstable $\ell=1, k=18$ mode, in terms of the mass coordinate, corresponding to our $0.585 M_{\odot} \mathrm{DB}$ template model analyzed in Fig. 3. $\mathrm{d} W / \mathrm{d} r$ is in arbitrary units. The gray regions show the locations of the convection zones. The internal chemical abundances of helium, carbon, and oxygen are also shown.

In summary, the excitation of the overstable $k=18 g$-mode of our template DB model is not affected by the presence of C at $-\log \left(1-M_{r} / M_{*}\right) \sim 8-9$. Since this property is shared by all the overstable $g$-modes of the DB models considered in this work, we conclude that overstable $g$-modes of DB models with thin He-rich envelopes, are excited in the same way as in DB models with thick He-rich envelopes. This property suggests that the DBV instability strip could be populated by DB white dwarfs with both thin and thick He-rich envelopes. This possibility could be tested by a asteroseismological analysis performed on well-studied DBV stars.

The details of the driving/damping process in our hot DQ template model for a selected overstable dipole mode with $k=18$ and $\Pi=847 \mathrm{~s}$ is displayed in Fig. 5. This mode, which is indicated by a circumscribed dot in the lower curve of Fig. 3, has the highest growth rate in our DQ template model $\left(\eta=9 \times 10^{-7}\right)$. We note that, at variance with the DB template model, most excitation originates in the C-bump, located at $-\log \left(1-M_{r} / M_{*}\right) \sim 9.5\left(T=1.177 \times 10^{6} \mathrm{~K}\right)$, close to the bottom of the outer convection zone. We caution, however, that some of the driving in this region could be caused by neglecting the perturbation of the convective flux (the frozenconvection approximation), as assumed in this work, instead of "genuine" driving due to the $\kappa$-mechanism acting at the C-bump. We would therefore expect the driving in this region to be somewhat lower if the frozen-convection assumption were relaxed in the stability computations. There are two additional driving regions, which also contribute to the mode excitation, located at $-\log \left(1-M_{r} / M_{*}\right) \sim 10.5$ and $\sim 12$, respectively. The most external one is located on the hot side of the opacity bump of the partial ionization of HeII, corresponding to $-\log \left(1-M_{r} / M_{*}\right) \sim 13$ and $T=7.14 \times 10^{4} \mathrm{~K}$. On the other hand, the presence of the driving region at $-\log \left(1-M_{r} / M_{*}\right) \sim 10.5$ is not directly 


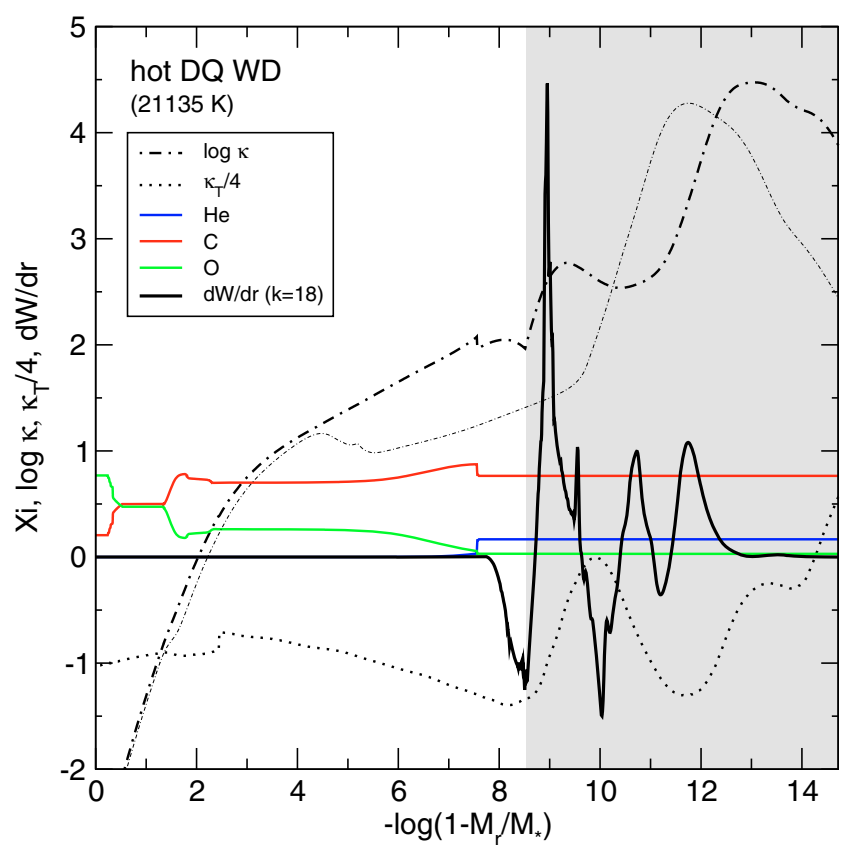

Fig. 5. Same as Fig. 4, but for our $0.585 M_{\odot}$ DQ template model analyzed in Fig. 3. The overstable mode has $\ell=1, k=18$ and $\Pi=847 \mathrm{~s}$.

associated with any bump in the opacity. This is an absent feature in the models of Fontaine et al. (2008). There are also three regions in our model that contribute to the damping of the mode, instead of two regions for the models of Fontaine et al. (2008). The total driving produced by the excitation regions overcomes the damping effects, and the mode is globally unstable.

The characteristics of the driving/damping process described above for a particular overstable mode of our DQ template model are also valid for all the unstable modes in all our hot DQ models. Thus, we conclude that overstable $g$-modes in hot DQ white dwarf models are primarily driven by the strong destabilizing effect of the opacity bump produced by the partial ionization of $\mathrm{CV}$ and CVI in a way similar to what occurs in GW Vir stars (Córsico et al. 2006), the partial ionization of HeII having a far less prominent role. These results are consistent with those reported by Fontaine et al. (2008), although in their models the partial ionization of HeII plays a more relevant role in the mode driving.

\subsection{The theoretical blue edges}

Since spectroscopic measurements of white dwarfs provide effective temperatures and surface gravities, it is useful to see how the evolutionary tracks and the instability domains look on the $T_{\text {eff }}-\log g$ plane. In Fig. 6, we plot a set of DB evolutionary sequences on this plane (thin solid lines), extracted from the nonadiabatic study of DBVs of Córsico et al. (2009). These sequences correspond to DB white dwarf models characterized by thick He-rich envelopes $\left(M_{\mathrm{He}} \sim 10^{-3} M_{*}\right)$, as a result of which, they never become DQ white dwarfs. We superimpose two evolutionary tracks $\left(M_{*}=0.585 M_{\odot}\right.$ and $\left.0.870 M_{\odot}\right)$ corresponding to DB white dwarfs with thin He-rich envelopes (thick solid curves). We note that, for a given value of the stellar mass, the tracks for DB models with thin and thick He-rich envelopes do not differ appreciably.

Figure 6 includes the location of known DBVs - extracted from Beauchamp et al. (1999) and Nitta et al. (2009) - and also the three known variable DQ white dwarfs (hereafter "DQVs").

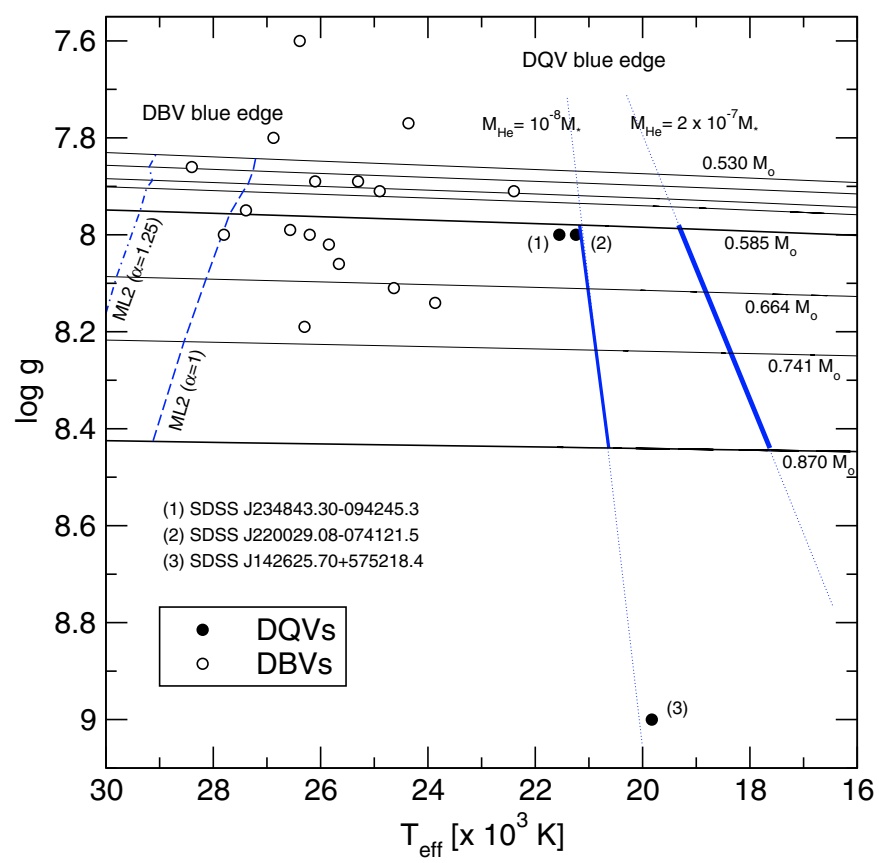

Fig. 6. A $\log g-T_{\text {eff }}$ diagram showing the location of the three known variable DQs (the "DQVs") with filled circles, and the known seventeen DBVs (hollow circles). Evolutionary tracks corresponding to DB white dwarf models with stellar masses of $0.530,0.542,0.556,0.565,0.589$, $0.664,0.742$, and $0.87 M_{\odot}$ (from up to down) are depicted by solid curves. The blue edge of the theoretical DBV instability strip - taken from Córsico et al. (2009) - is displayed with blue dashed lines. The blue edge of the DQV instability domain (present work) is also drawn with thick blue solid segments for two values of $M_{\mathrm{He}}$. For illustrative purposes, these lines are extended to high and low gravities (thin blue dotted lines).

We note that the observed blue edge for the DBV instability strip is accounted for well by a theoretical blue edge from nonadiabatic computations on DB models with the ML2 ( $\alpha=1$ or $\alpha=1.25$ ) flavor of the mixing length theory (MLT) of convection (Córsico et al. 2009). We also display in the figure the location of the blue edge for the DQV instability domain (thick solid segments). We recall that our DB and DQ white dwarf models assume the ML2 $(\alpha=1)$ prescription of the MLT. We found that our DQ white models become pulsationally unstable shortly after their formation, as can be seen by comparing the blue edge of DQVs from Fig. 6 with the values of effective temperature at which DQ white dwarf models are formed (see Table 1 of Althaus et al. 2009).

A important feature of Fig. 6 is that, in the current computations, the dependence of the blue edge on the stellar mass of the DQVs has an opposite sense to that found for the DBVs, that is, we find that the blue edge is hotter for less massive DQV stars. This is because, in the context of the diffusive/convective mixing scenario, the temperature at which the DQV blue edge appears depends directly on both the stellar mass and the thickness of the He-rich envelope of the DB white dwarfs from which the DQ stars are formed. In particular, we have shown in Sect. 3 (see Fig. 2) that for the He envelope masses assumed in our models, the merger of the two convective zones, and thus, the formation of the DQ white dwarfs, occurs at higher effective temperatures for the less massive models than for the more massive ones. Since the DQV blue edge occurs at the $T_{\text {eff }}$ at which the DQs are formed, this blue edge is then hotter for less massive models. We note that this trend is at odds with the results of the stability 


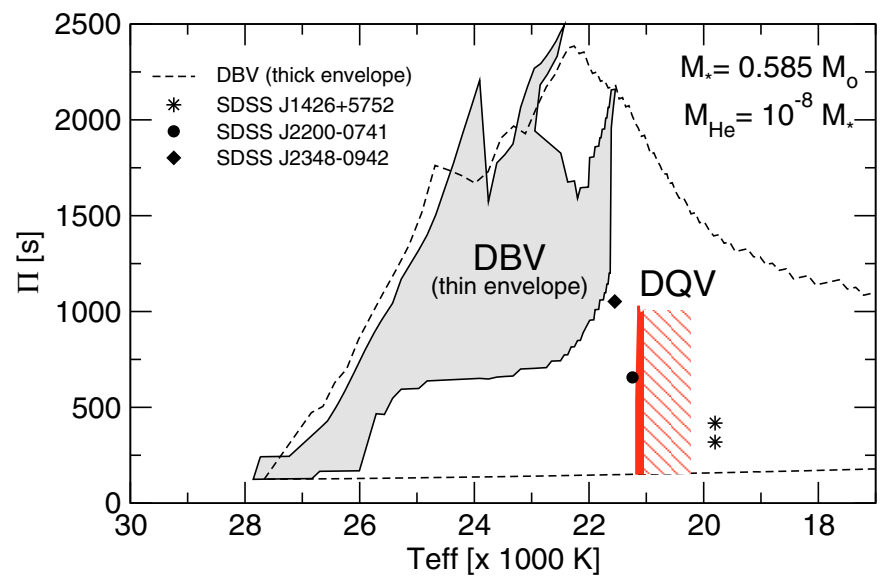

Fig. 7. The instability domains on the $T_{\text {eff }}-\Pi$ plane for $\ell=1 g$-modes corresponding to our set of models with $M_{*}=0.585 M_{\odot}$ and $M_{\mathrm{He}}=$ $10^{-8} M_{*}$

analysis of Fontaine et al. (2008) and also the order-ofmagnitude estimation obtained by Montgomery et al. (2008) from the thermal timescale $\left(\tau_{\text {th }}\right)$ at the base of the outer convection zone.

Figure 6 also shows that the blue edge is hotter (2000-3000 K) for DQVs with smaller amounts of He. This is because the transition of a DB to a DQ white dwarf occurs early when the He envelope of the DB model is less massive, as explained in Althaus et al. (2009). Since the pulsations are excited immediately after the formation itself of DQs, the blue edge for DQV stars is hotter for smaller amounts of $\mathrm{He}$ at the envelope.

We close this section by noting that the location of the three DQV stars in the $T_{\text {eff }}-\log g$ plane is qualitatively consistent with the results of our computations for the blue edge when $M_{\mathrm{He}} / M_{*}=10^{-8}$. Only SDSS J142625.70+575218.4 is located at right of this theoretical blue edge. We note that this star has a surface gravity large in excess, and no available track passes close to its location. Presumably, this star has a stellar mass $\sim 1.2 M_{\odot}$. The other two DQVs, SDSS J220029.08-074121.5 and SDSS $\mathrm{J} 234843.30-094245.3$, with a stellar mass close to $0.6 M_{\odot}$, are located at temperatures slightly higher than the predicted blue edge. We note, however, that the blue edge could be easily accommodated at higher effective temperatures by simply considering stellar models with somewhat thinner He-rich envelopes $\left(M_{\mathrm{He}} / M_{*} \lesssim 10^{-8}\right)$.

\subsection{The $T_{\mathrm{eff}}-\Pi$ plane}

We explore the domains of unstable dipole modes in terms of the effective temperature. Figure 7 shows the instability domains on the $T_{\text {eff }}-\Pi$ plane for the sequence of $M_{*}=0.585 M_{\odot}$ and $M_{\mathrm{He}}=10^{-8} M_{*}$. The DBV instability domain is shown as a gray area, whereas the beginning of the DQV instability region is indicated by a vertical red strip. In the plot, we also include the periods detected in the three known DQV stars. In the interests of a comparison, we also include the instability domain of DB models with thick He-rich envelopes $\left(M_{\mathrm{He}} \sim 1 \times 10^{-3} M_{*}\right)$, according to Córsico et al. (2009) (dashed lines). Figures 8-10 depict the situation for the remaining sequences considered in this work. In each figure, we have drawn a striped region to illustrate the possible extension to lower temperatures of the DQV instability domains.

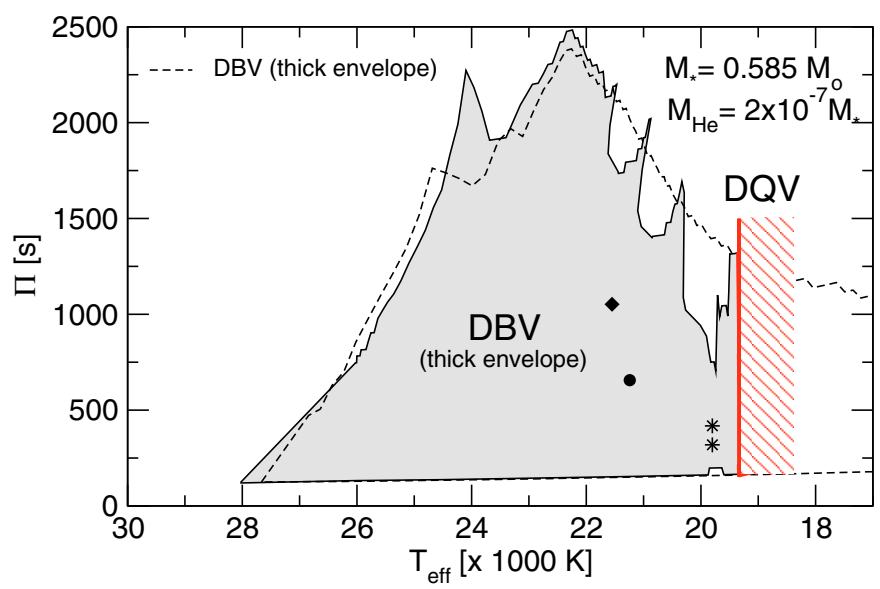

Fig. 8. Same as Fig. 7, but for models with $M_{\mathrm{He}}=2 \times 10^{-7} M_{*}$.

To begin with, we note that the hot side of the DBV instability domain for thin He-rich envelope models is virtually identical to the case of DB models with thick He-rich envelopes. We already mentioned this feature in Sect. 4.1. This means that DBVs with thick $\left(M_{\mathrm{He}} \sim 10^{-3} M_{*}\right)$ or thin $\left(M_{\mathrm{He}} \sim 10^{-7}-10^{-8} M_{*}\right)$ He-rich envelopes should exhibit the same ranges of excited periods, at least in the hot half of the instability domain. Both types of DBV stars have the following properties. Firstly, there is a strong dependence of the longest excited periods on the stellar mass, such that the periods are longer for less massive models. Secondly, the shorter excited periods, in contrast, do not exhibit any dependence on $M_{*}$. Finally, the blue edge is hotter for more massive DB models.

An interesting feature displayed by the plots is that the instability domains of DBVs and DQVs are clearly separated in models with $M_{\mathrm{He}}=10^{-8} M_{*}$ (Figs. 7 and 9). There is a noticeable gap in effective temperature between the point at which DB pulsations no longer exist and the location of the blue edge of the DQV instability strip, in which the pulsations of DQs begin. This gap is more pronounced for white dwarfs with $M_{*}=0.870 M_{\odot}\left(\Delta T_{\text {eff }} \sim 1200 \mathrm{~K}\right)$ than for white dwarfs with $M_{*}=0.585 M_{\odot}\left(\Delta T_{\text {eff }} \sim 400 \mathrm{~K}\right)$. For models with $M_{\mathrm{He}}=2 \times 10^{-7} M_{*}$, on the other hand, the instability domains are "in contact" (Figs. 8 and 10). Thus, there exists a continuous transition from the pulsation instability of DBs to DQs. We recall, however, that modes in DBs are excited by the maximum in opacity caused by the partial ionization of He, whereas overstable modes in DQs are mainly driven by the opacity bump produced by the partial ionization of $\mathrm{C}$.

In Figs. 7 to 10, we included the periods detected in the three presently known DQV stars. The longest periods excited in our DQ models are in the range $750-1500 \mathrm{~s}$. The periods detected in SDSS J142625.70+575218.4 and SDSS J220029.08-074121.5 are well within the predicted period ranges, but the period at $\Pi \approx 1052 \mathrm{~s}$ corresponding to SDSS $\mathrm{J} 234843.30-094245.3$ is too long and is not represented well by our models, except when $M_{*}=0.585 M_{\odot}$ and $M_{\mathrm{He}}=$ $2 \times 10^{-7} M_{*}$. On the other hand, only the sequences of DQs with $M_{\mathrm{He}}=10^{-8} M_{*}$ are able to nearly account for the effective temperatures at which the variable DQ stars are located. As we mentioned in Sect. 4.2, we could apparently remove the small discrepancies in $T_{\text {eff }}$ simply by considering smaller amounts of $\mathrm{He}$ in the envelopes. 


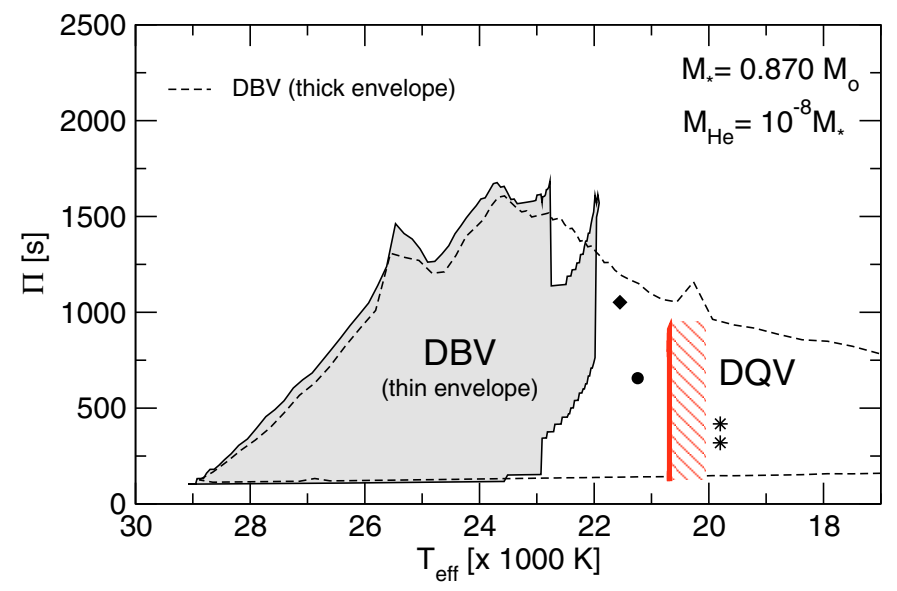

Fig. 9. Same as Fig. 7, but for models with $M_{*}=0.870 M_{\odot}$.

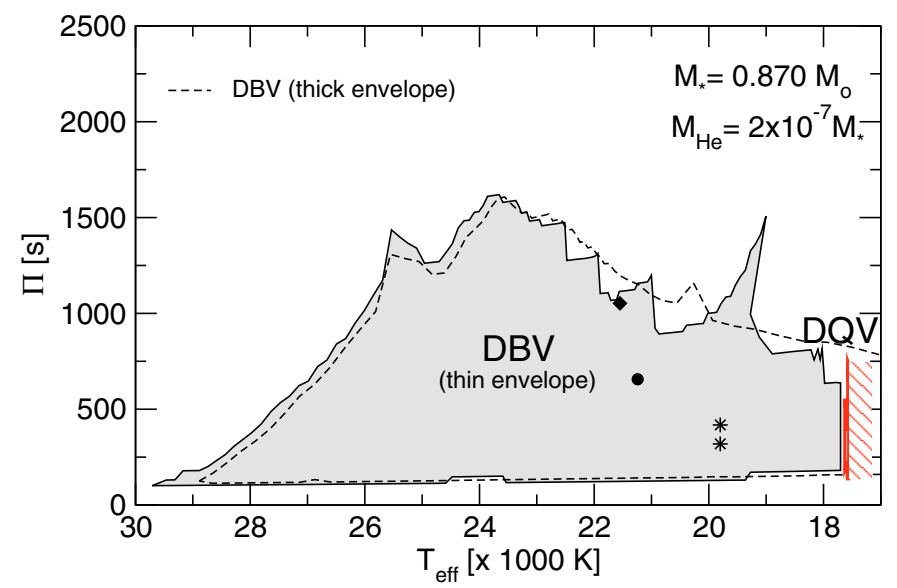

Fig. 10. Same as Fig. 7, but for models with $M_{*}=0.870 M_{\odot}$ and $M_{\mathrm{He}}=$ $2 \times 10^{-7} M_{*}$.

\section{Summary and conclusions}

In this study, we have presented a pulsation stability analysis of carbon-rich hot DQ white dwarf stars. These stars constitute a new class of white dwarf stars, uncovered within the framework of SDSS project. There exist nine hot DQs, out of a total of several thousands white dwarfs spectroscopically identified. Three hot DQ white dwarfs have been found to exhibit photometric variations with periods compatible with pulsation $g$-modes. One of the main aims of the present work was to test the convectivemixing picture for the origin of hot DQs by studying their pulsational properties. Specifically, we have employed the full evolutionary models of hot DQ white dwarfs developed by Althaus et al. (2009), which consistently cover the entire evolution from the born-again stage to the DQ white dwarf stage. We have presented a stability analysis on white dwarf models from stages before the blue edge of the DBV instability strip $\left(T_{\mathrm{eff}} \approx 30000 \mathrm{~K}\right)$ until the domain of the hot DQ white dwarfs (18 000-24000 K), including the transition from DB to hot DQ white dwarfs. We have explored evolutionary models with $M_{*}=0.585 M_{\odot}$ and $M_{*}=0.87 M_{\odot}$, and two values for the thickness of the He-rich envelope $\left(M_{\mathrm{He}}=2 \times 10^{-7} M_{*}\right.$ and $\left.M_{\mathrm{He}}=10^{-8} M_{*}\right)$. These envelopes are 4-5 order of magnitude thinner than those of standard DB white dwarf models derived from canonical stellar evolution calculations.
We found that at epochs when the models are characterized by He-dominated atmospheres, they exhibit $g$-mode pulsations typical of DBV stars, and when the models become hot DQ white dwarfs with carbon-dominated atmospheres, they continue being pulsationally unstable with characteristics similar to those of DB models, and with periods in qualitative agreement with those observationally detected in variable hot DQ white dwarfs. Our main results are the following:

- In general, $g$-modes excited in hot DQ white dwarfs have low linear growth rates, noticeably lower than those typical of DB models. In spite of this, the excited modes in hot DQ white dwarfs should have sufficient time to reach observable amplitudes. This conclusion is in line with the results of Fontaine et al. (2008).

- Overstable $g$-modes of DB white dwarf models with thin He-rich envelopes $\left(M_{\mathrm{He}} / M_{*} \sim 10^{-8}-10^{-7}\right)$ are excited in the same way as in DB models with thick He-rich envelopes $\left(M_{\mathrm{He}} / M_{*} \sim 10^{-3}\right)$, that is, by the action of the $\kappa$-mechanism acting on the region of partial ionization of HeII. The opacity bump caused by the partial ionization of $\mathrm{C}$, present in DB models with thin envelopes, does not contribute at all to the destabilization of modes.

- Overstable $g$-modes in hot DQ white dwarf models are primarily driven by the $\kappa$-mechanism caused by the strong destabilizing effect of the opacity bump due to the partial ionization of $\mathrm{C}$, and the role of the partial ionization of HeII is far less relevant. This is slightly inconsistent with the models of Fontaine et al. (2008), which predict that both opacity bumps are equally important in destabilizing of modes.

- The blue edge of DQVs is hotter for less massive models than for more massive ones, which is at odds with the results of Fontaine et al. (2008) and Montgomery et al. (2008). The reason for this discrepancy relies on the way in which the DQ models are conceived, which in our case concerns the result of fully evolutionary computations in the context of the diffusive/convective mixing scenario presented by Althaus et al. (2009).

- The blue edge for DQV stars is hotter for smaller amounts of $\mathrm{He}$ in the envelopes.

- The location of the three DQV stars in the $T_{\text {eff }}-\log g$ diagram is qualitatively accounted for by our calculations of the blue edge with $\log \left(M_{\mathrm{He}} / M_{*}\right)=-8$, and the small discrepancies in $T_{\text {eff }}$ could be accounted for by simply considering stellar models with somewhat thinner He-rich envelopes $\left(\log \left(M_{\mathrm{He}} / M_{*}\right) \lesssim-8\right)$.

- The prototype star SDSS J142625.70+575218.4, which lies to right of our theoretical DQV blue edge, has a surface gravity large in excess, and we have no available DB/DQ track that passes close to its location. Presumably, this star could have a stellar mass $\gtrsim 1.1 M_{\odot}$.

- The instability domains of DBVs and DQVs are clearly separated in the $T_{\text {eff }}-\Pi$ plane for models with the thinnest He-rich envelopes considered in this work $\left(M_{\mathrm{He}}=10^{-8} M_{*}\right)$, but there is a continuous transition between the pulsation instability of DBs and DQs for the case in which $M_{\mathrm{He}}=$ $2 \times 10^{-7} M_{*}$.

- The periods detected in SDSS J142625.70+575218.4 and SDSS J220029.08-074121.5 are well within our theoretical ranges of excited periods, but the period at $\Pi \approx 1052$ s corresponding to SDSS J234843.30-094245.3 is excessively long, and is not explained well by our models.

In summary, our calculations support the diffusive/convective mixing picture for the formation of hot DQs, an idea originally 
proposed by Dufour et al. (2008) and quantitatively elaborated on by Althaus et al. (2009). In particular, the results of the present work demonstrate that the diffusive/convective mixing scenario is not only able to explain the origin of hot $D Q$ white $d$ warfs, but also accounts for the variability of these stars.

According to this scenario, a fraction of DB white dwarfs those that are immediate progenitors of hot DQ white dwarfs should be characterized by He-rich envelopes $10^{3}$ to $10^{4}$ times thinner than the canonical thickness predicted by the standard theory for the formation of DB white dwarfs (Althaus et al. 2005). The existence of these DBs, and thus, the validity of the diffusive/convective mixing scenario, could be investigated using an adiabatic asteroseismological analysis of DBV stars with a rich pulsation spectrum and high quality observational data available, such as GD 358, EC 20058-5234, and CBS 114.

We also emphasize that the conclusions reached in this work - and also the results of Fontaine et al. (2008) and Dufour et al. (2008) - in particular concerning the location of the blue edge of the DBV and DQV instability strips, could be altered substantially if a fully consistent treatment of the interaction between convection and pulsation, such as that of Dupret et al. (2008) or even the simpler approach of Wu \& Goldreich (1999), were included in the stability analysis.

Acknowledgements. We would like to warmly acknowledge the comments and suggestions by our referee, Dr. M. H. Montgomery, that allowed us to improve the original version of this paper. This research was supported by AGENCIA: Programa de Modernización Tecnológica BID 1728/OC-AR, by the AGAUR, by MCINN grant AYA2008-04211-C02-01, by the European Union FEDER funds and by PIP 6521 grant from CONICET. LGA also acknowledges AGAUR through the Generalitat de Catalunya for a PIV grant. This research has made use of NASA's Astrophysics Data System. Finally, we thank H. Viturro and R. Martínez for technical support.

\section{References}

Althaus, L. G., Serenelli, A. M., Panei, J. A., et al. 2005, A\&A, 435, 631

Althaus, L. G., García-Berro, E., Córsico, A. H., et al. 2009, ApJ, 693, L23

Barlow, B. N., Dunlap, B. H., Rosen, R., et al. 2008, ApJ, 688, L95

Beauchamp, A., Wesemael, F., Bergeron, P., et al. 1999, ApJ, 516, 887

Bergeron, P., Legget, S. K., \& Ruiz, M. T. 2001, ApJS, 133, 413

Brassard, P., \& Fontaine, G. 1999, Theory and Tests of Convection in Stellar Structure, 173, 329

Burgers, J. M. 1969, Flow Equations for Composite Gases (New York: Academic Press)

Cassisi, S., Pothekin, A. Y., Pietrinferni, A., Catelan, M., \& Salaris, M. 2007, ApJ, 661, 1094

Córsico, A. H., Althaus, L. G., \& Miller Bertolami, M. M. 2006, A\&A, 458, 259 Córsico, A. H., Althaus, L. G., Miller Bertolami, M. M., et al. 2009, J. Phys. Conf. Ser., 172, 12075

Dufour, P., Liebert, J., Fontaine, G., \& Behara, N. 2007, Nature, 450, 522

Dufour, P., Fontaine, G., Liebert, J., Schmidt, G. D., \& Behara, N. 2008, ApJ, 683, 978

Dufour, P., Liebert, J., Swift, B., Fontaine, G., \& Sukhbold, T. 2009, J. Phys. Conf. Ser., 172, 12012

Dupret, M. A., Quirion, P. O., Fontaine, G., Brassard, P., \& Grigahcéne, A. 2008, J. Phys. Conf. Ser., 118, 012051

Fontaine, G., Brassard, P., \& Dufour, P. 2008, A\&A, 483, L1

Fontaine, G., Brassard, P., Dufour, P., Green, E. M., \& Liebert, J. 2009, J. Phys. Conf. Ser., 172, 12066

Haft, M., Raffelt, G., \& Weiss, A. 1994, ApJ, 425, 222

Iglesias, C. A., \& Rogers, F. J. 1997, ApJ, 464, 943

Itoh, N., Hayashi, H., Nishikawa, A., et al. 1996, ApJS, 102, 41

Lee, U., \& Bradley, P. A. 1993, ApJ, 418, 855

Magni, G., \& Mazzitelli, I. 1979, A\&A, 72, 134

Miller Bertolami, M. M., \& Althaus, L. G. 2006, A\&A, 454, 845

Montgomery, M. H., Williams, K. A., Winget, D. E., et al. 2008, ApJ, 678, L51

Nitta, A., Kleinman, S. J., Krzesinski, J., et al. 2009, ApJ, 690, 560

Segretain, L., Chabrier, G., Hernanz, M., García-Berro, E., \& Isern, J. 1994, ApJ, 434,641

Tassoul, M., Fontaine, G., \& Winget, D. 1990, ApJS, 72, 335

Unno, W., Osaki, Y., Ando, H., Saio, H., \& Shibahashi, H. 1989, Nonradial Oscillations of Stars (University of Tokyo Press), 2nd edn. 